\title{
Sustainable housing maintenance management model
}

\author{
A. A. Olanrewaju, T. S. Yeow \& L. L. Tat \\ Department of Construction Management, \\ Faculty of Engineering and Green Technology, \\ Universiti Tunku Abdul Rahman, Malaysia
}

\begin{abstract}
The drive towards sustainable development is achievable through the maintenance of the existing housing stock. But this is constrained by the lack of a connective link between maintenance management and sustainable development. This lack of connection can be explained by the poor perception and understanding of the scope of maintenance. Based on a synthesis of literature and discussions with experts, a conceptual sustainable housing maintenance management model that would allow sustainable development to be set as the objective for maintenance was presented. Not only does the model does show that sustainable development can be set as the maintenance objective, but both sustainable and maintenance requirements can be assimilated into management theories and practices. The key variables in sustainable housing maintenance management can be categorized into planning, controlling, organizing, directing and implementing functions. The study, though in the initial stage, represents a potential contribution to sustainable maintenance management for use in the built environment.
\end{abstract}

Keywords: sustainability, residential buildings, housing operation, rethinking sustainable, maintenance model.

\section{Introduction}

Research on building maintenance is not lacking, though various studies use different approaches. However, the addition of the term 'sustainable' to the 'maintenance' concept changes it meaning and scopes. This new concept or definition means that the scope of maintenance must not only focus on increasing the building performance but should also consider the implications of maintenance 
or the lack of it on sustainable development. There is a fundamental need to consider sustainable development in maintenance, because the processes and procedures involves in maintenance management have great impact on social, economic and environmental issues. Whenever you make a choice to or not to maintain your building, you are contributing to the carbon emission - you leave a carbon footprint! Simply put, you are part of the obstacles to sustainable development. Because you consumed resourcing including electricity, gas and use water - you are destroying the environment, and economic and communities' values are tampered with. You can avoid or lessen these with the way you manage your maintenance. The amount of global warming caused by housing maintenance activities and its effects are uncertain but what is certain is that the amount is on the increase unabated. This poses risk and a blink future to everyone.

Towards sustainable development, housing remains a strategic product, as housing and the services it provides remain necessary to propelled economic prosperity, social development and optimizes the rate at which natural resources are depleted and reduces degradation of natural resources. However, the requirements to situate sustainable development in housing maintenance are making the roles of maintenance organizations more complex and sophisticated with them taking over unfinished construction or projects that do not meets with sustainability requirements, yet are mandated to provide sustainable maintenance. This will still be the case even if all buildings meet with the requirements of sustainable development, as building age and decay throughout their life span. Furthermore, while standards and regulations are becoming available for sustainable construction, the same is not available for sustainable maintenance. Although new 'sustainable housing' is encouraged, it must exist alongside the current housing stock and infrastructure that does not comply with the requirements of sustainable development. While populations are projected to increase significantly, the rate of building replacement is very low. In Malaysia for instance, this is less than $1 \%$. This has two immediate implications. First, the existing housing stock though not designed / built to comply with sustainable requirements which though are more than $90 \%$ of the total stock will be available in the next 50 to 100 years. The second implication is there will be increased in maintenance demand. This is because a major cause of housing maintenance is the number of users and their behaviours.

In general, the sustainability criteria are divided into two; quantitative and qualitative criteria. The quantitative aspects had received (and are receiving) considerable attention. The quantitative aspect involves, using energy saving materials and components in construction of the buildings and design layout that would save energy and water, promote social interaction, healthy living styles, securities and environmentally friendly designed. However, it is tempting to think that sustainable development is just a function of the quantitative criteria. It is argued that the qualitative criteria are the most significant.

This study applies the general management theory to explore the conceptual foundation and nature of sustainable housing maintenance. Hence, will like to stress that a question that the study aim examine is what significance does 'management theory' offers to sustainable maintenance, if each of the functions is 
consider separately? The value that management has on sustainable maintenance includes; determine maintenance objectives, establish strategic objectives, set maintenance standards, determine maintenance performance indicators, identify user and their value systems, set priorities, reduce 're-maintenance', deliver better service and maximizes productivity and profits. In order to achieve the above objectives, there is a need to correctly articulate sustainable maintenance into a management theory and practice that systematically focuses on various aspects to add 'value to money'.

Some authors have addressed maintenance planning (see for instance: Mills [1], Chanter and Swallow [2], Wood [3]). With specific reference to sustainable maintenance, Jones and Cooper [4] have sorted the facts to know the extent sustainability issues are considered in the development of maintenance plan. But such studies do not provide the systemic and holistic link the planning function has with the other management functions. Therefore, to contribute to knowledge to this area, this paper reports a study that provides sustainability as the objective for housing maintenance management. However, to facilitate using maintenance as a platform to manage sustainable criteria in the maintenance of existing housing stock, a sustainable housing maintenance management model, or SHM3 ${ }^{\circledR}$ was developed.

\section{Sustainable housing maintenance management}

Many authors and researchers have defined maintenance (see for example: Jones and Sharp [5] and Wood [3]). These definitions are obstacles towards allowing maintenance to contribute to sustainable development, since maintenance is not initiated based on user value systems rather the building conditions. Or at best these understanding or definitions would only account for the engineering or technical aspects of sustainability. Addressing quantitative performance criteria only without homeostatic consideration of qualitative performance criteria (e.g. the cultural and behavioural aspect of the building occupants and maintenance organizations) is grossly inadequate (see also Chanter and Swallow [2]). Despite the advancement in technology, government regulations and edicts, significance changes have not been achieved in adopting sustainable maintenance practices and strategies. Anecdotal evidence has suggested that technical aspect is attributable to about $40 \%$ performance while the remaining $60 \%$ is for qualitative criteria.

Here, maintenance is defined as the required processes and services to preserve, protect, improve and care for a building's fabric, structure and engineering services after completion, refurbishment or replacement to current standards to enable it meet the user's value systems throughout its entire lifespan without drastically upsetting its basic (physical) forms and use. The definition seems to be long [3], but meaningful. It portends the reality, because in practice maintenance should optimize the users/clients' value system. To this end, maintenance is considered in terms of three requirements, namely physical, technological requirements and cultural and behavioural issues of the stakeholders. If sustainability is the major criterion within the user value system, it will be set as the main maintenance objective. 
Maintenance is a strategic to building performance and occupants' productivity and satisfactions and the performance of the housing and occupant's satisfactions have far reaching impact on sustainable development and national prosperity. A building that does not meet the required maintenance standards will certainly produce more waste and pollutants (e.g. toxic build-up, carbon dioxide emission), affect users' well-being as well as consume more energy, water and other resources [6]. It is arguable that perhaps more than the design team, the maintenance management aspect has more roles to play in achieving sustainable housing. The lifespan of buildings is high (i.e. 100 years), during which they consume energy and water and release $\mathrm{CO}_{2}$. Therefore, if residential housing are maintain to reduce energy and water consumptions and curtail carbon emission, enhance occupants' productivity, this will save million metric tons of $\mathrm{CO}_{2}$, and addition to increase well-being and total user values systems. At the design and construction stages only the technical sustainable criteria can be inculcated into the building. On their own right this aspect is required and should be encouraged. But there is the qualitative aspect - the cultural and behavioural aspect of the building users and maintenance organizations.

To understand the importance of maintenance towards sustainability targets, international recognized systems for assessing buildings performance, including LEED (Leadership in Energy and Environmental Design), BREEAM (Building Research Establishment Environmental Assessment Method), Greenstar (Australia), Sustainable Building Certificate (Germany), Haute Qualite Environment (France), Green Building Index (Malaysia) consider maintenance issues in their assessment criteria. But, like government regulations and laws, these third-party tools are more appropriate to new project but not for maintenance.

Sustainable building provides energy saving, water saving, healthier indoor environments, better connectivity to social facilities and amenities (public transport) while embracing resource recycling initiatives. The need for maintenance in buildings will only intensify as the value of the building and the associated engineering services must be preserved and sustained for the building to be meaningful to its users throughout its life span. Basically, there is no way existing housing can comply with the sustainable agenda without maintenance the correlation between maintenance and sustainability is positive. A variable that is common to both sustainable development and maintenance is that both are future based. To illustrate this simply, the primary essence of maintenance is to enable the building to meet user value system throughout it the building life span (reader may examine the definition we provided above for maintenance).Sustainable development and maintenance, both being tactical or technical issues, requires management to blend the requirements in both to deliver best value added services or functions to develop new set of objectives and purposes (Figure 1).

These interdependencies are what will be further explored. While studies on the actual impact of maintenance on sustainable developments are not conclusive [7], a number of researchers have proved that maintenance has an impact on sustainable development [4, 8] and identified various sustainable factors in maintenance practice. These factors include lifecycle approach, the 3Rs of 


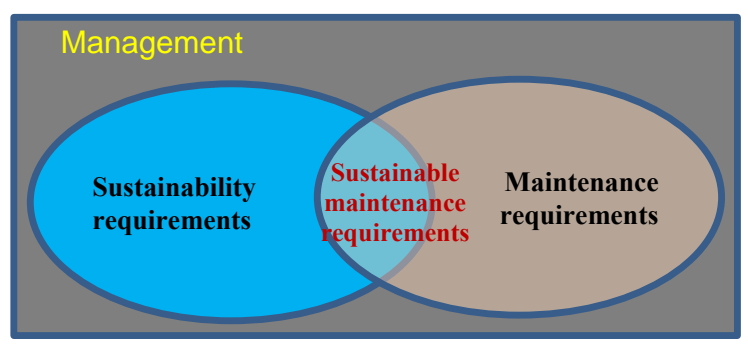

Figure 1: Simple sustainable maintenance management framework.

material and components, to use green technologies, using low embodied materials and components, integrated multidisciplinary approach and to use local materials. With specific reference to housing maintenance, it was found that it is possible to reduce maintenance cost by taken into account factors that affect operational costs [9]. But the authors only consider maintenance cost at design and construction stages. There are also studies on technological aspect of sustainable issues in the housing stock (see also Zhu and Lin [9] and Swan et al. [11]). However, these aspects, has no much to do with the users' and maintenance organizations' culture and behaviours. But, these aspects have no sustainability values except the users have positive attitudes towards them [12]. Users/organizations' culture /behaviours is the strategic factor that drives the achievement of sustainable development. Maintenance culture is the main ingredient towards a successful sustainable maintenance management. Occupants /organizations with positive maintenance and sustainability culture will have positive attitudes towards the framework. To make this explicit, management theory need to be applied to examine and consider the requirements of both the occupants and maintainers. In general, a significant aspect of the housing users' and maintenance managers' understanding of the sustainable maintenance cost is limited to a narrow scope and benefits of the operational aspects and specifically to repair and corrective maintenance.

Such poor perception of maintenance will remain if the maintenance demand is based on the building rather the users. However, if maintenance and its management are not redefined, all the various functions in management will be inconsistent in the process chain, as the importance of maintenance in sustainable development would not be clear and optimized. For sustainable maintenance to be seen as value added service, the requirements of both theories and practices should be matched into the management framework. Based on the current practice, much focuses has been on maintainability rather than on maintenance management. This deficiency is prevalent because sustainable maintenance is not position in management theory and practices but rather limited in scope to one aspect/function of management. Contrast against the problems illustrated above, this study situates sustainable maintenance into the management theory and practice.

Based on a comprehensive review of literature and a brief discussion with selected members of CIOB Malaysia, recently (September 2014), on the relationship between sustainability development and housing maintenance, a sustainable housing maintenance management model SHM $3^{\circledR}$ was proposed for 
inculcating sustainable issues in the management of housing, as shown in Figure 2. The SHM $3^{\circledR}$ consists of five major partitions of: planning, controlling, organizing, directing and implementing. This provides a step by step methodology for maintenance demand and implementation. With this sequential arrangement, maintenance is strategically and consistently planned, organized, directed, controlled and executed. In other words, maintenance is considered an investment that contributes to an organization's corporate business objectives.

A critical stage in the model is the 'maintenance objectives-mission statement'; at this stage sustainable development will be set as the maintenance objective. It is on this basis that other sub-functions in the planning stage will stem from. This subsequently forms the basis of other functions in the management theory and practice. As it can be seen under this model, implementation is not the 'beginning' and planning is not to be treated in isolation.

During the discussion with the CIOB members, it was confirm that since the clients/occupants are those to benefits more in the long run with sustainable maintenance management, it is thereby imperative that the clients and users should demand for it, to be willing and ready to pay the costs. However, we argued that, the era of the clients asking the industry for best practice is over, the industry needs to lead. It is the failure of the industry to apportion blame to clients, whose more than $90 \%$ are one-off! The clients need the industry to provide value for money services and products. Furthermore, it was observed that the maintenance and facilities managers have poor understanding on the scope and meaning of maintenance, maintenance management and sustainable development. This is compounded by the lack of interests by maintenance organizations promote sustainable maintenance management. Therefore, the industry should lead the clients to demand sustainable maintenance in order to realize values added to their investments. As the major regulator, the government should enforce in the way of regulations and laws to encourage the maintenance organizations towards sustainable maintenance management practices.

\section{Summary}

This paper seeks to prompt active discussion about what are important to the operators/managers of sustainable buildings. It is also obvious that the major problem in housing maintenance is not about the technology but rather about its management. This paper has drawn attention to some of the practical difficulties that exist within the definition and scope of maintenance thereby limiting it to serves as a platform to drives sustainable development but this due to poor perception and not reality. The original research question for this study revolved around whether considering one aspect of management is sufficient to define the scope and objective of sustainable maintenance. It is surmised that this is not sufficient in anyway. There is a need to consider all the various aspects of management in sustainable maintenance to achieve sustainable maintenance and consequently sustainable development. The owners of the housing stocks should demand for sustainable maintenance management in efforts to derive the best value on their investment. 


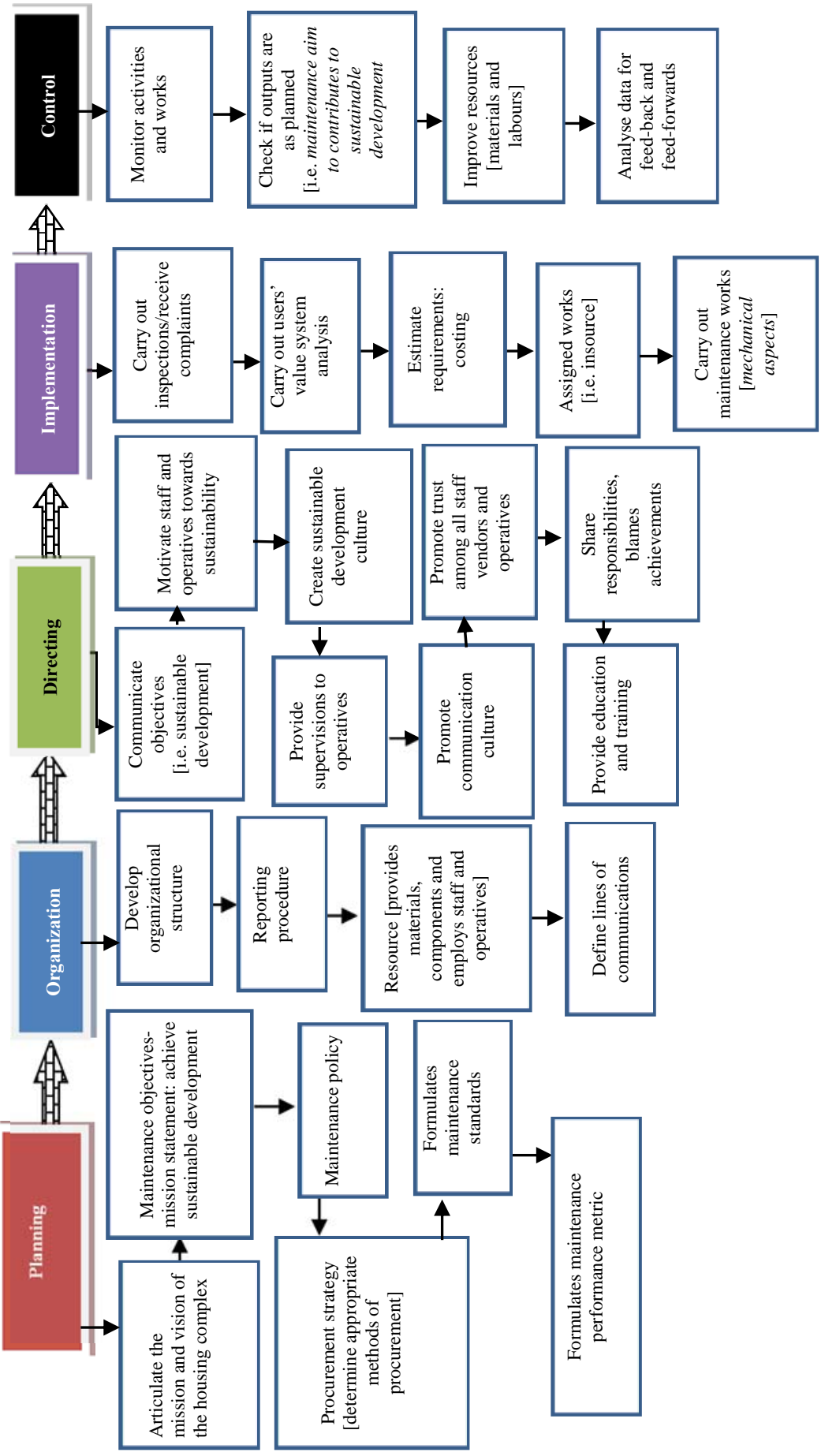

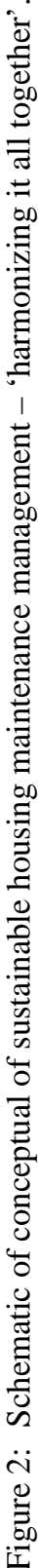


The SHM3 $^{\circledR}$ considers sustainability requirements as the motivation for maintenance demand. The prevailing justifications for this have been mentioned earlier. But to reiterate, it involve setting sustainable development as the target for maintenance, setting strategic objectives, identifying housing owner value system, providing consistency link with other functions, using both objectives and subjective criteria in housing maintenance decision practices, evaluating performance, enhancing communication, user-focused decision making, continuous improvements, encourage team work, and to comply with other guideline on sustainable development. SHM $3^{\circledR}$ is different from the current ways in which maintenance is managed, however, it put emphasis that the conventional maintenance management practices and processes followed but primary target should aim to reduce waste, minimise water usage, reduce carbon emission, enhances social and communities integration, reduce cost and total client/user value systems.

The model suggests that the maintenance management is linear and simple but in reality may be quite challenging. If there are complexities or uncertainties involved with certain functions or the maintenance organisations does not possess the required expertise to provide the required services, 'help' should be sought from those that have sufficient knowledge in sustainable maintenance practices.

\section{References}

[1] Mills, E. D. (ed.), Building maintenance and preservation; a guide to design and management, $2^{\text {nd }}$ ed. Oxford: Butterworth-Heinemann, Oxford, 1994.

[2] Chanter, B. \& Swallow, P., Building maintenance management, Blackwell Publishing, Oxford, 2007.

[3] Wood, B. R., Building maintenance. Oxford: Blackwell Publishing, 2009.

[4] Jones, K. \& Cooper, J., The role of routine maintenance in improving the sustainability of existing social housing. In Proceedings of the European Network for Housing Research W07 - Housing Regeneration and Maintenance. Sustainable Urban Areas, 25-23 June 2007 (Rotterdam 2007).

[5] Jones, K. \& Sharp, M., A new performance based process model for built asset maintenance. Facilities, Vol. 25, No. 13/14, pp. 525-535, 2007.

[6] Olanrewaju A. A \& Kafayah, S. T., The Need to maintain our buildings: Sustainable Development. In Proceedings of PSIS Enviro (The 1st National Seminar on Environment, Development and Sustainability). Politeknik Sultan Idris Shah, PSIS, Sabak Bernam Selangor Malaysia, 2008.

[7] Miller, W. \& Buys, L., Factors influencing sustainability outcomes of housing in subtropical Australia. Smart and Sustainable Built Environment, 2(1), pp. 60-83, 2013.

[8] Wood, B. R., The role of existing buildings in the sustainability agenda, Facilities, Vol. 24, No. 1/2, pp. 61-67, 2005.

[9] Zhu, Y. \& Lin, B., Sustainable housing and urban construction in China, Energy and Buildings, 36, pp. 1287-1297, 2004. 
[10] Stoy, C. \& Kytzia, S., Utility cost: a survey of Swiss office building, Journal of Facilities Management, 6(2), 120-131, 2008.

[11] Swan, W. Ruddock, L. Smith, R Fitton, R., Adoption of sustainable retrofit in UK social housing, “Structural Survey”, 31(3), 181-193, 2013.

[12] Jenks, M \& Jones, C. (eds), Dimensions of the sustainability city. Dordrecht: Springer, 2010. 